\title{
HELMINTIASIS EN ALPACAS (Vicugna pacos) DE DOS COMUNIDADES DE MACUSANI, PUNO, DURANTE LA ÉPOCA SECA
}

\author{
Helminthiasis in Alpacas (Vicugna pacos) of Two Peasant Communities in \\ Macusani, Puno during the Dry Season
}

\author{
Nancy Contreras S. ${ }^{1}$, Amanda Chávez V. ${ }^{1,4}$, Rosa Pinedo V. ${ }^{1}$, Víctor Leyva V. ${ }^{2}$, \\ Francisco Suárez A. ${ }^{3}$
}

\section{Resumen}

El estudio tuvo por objetivo estimar la prevalencia de helmintos gastrointestinales en alpacas de dos comunidades del distrito de Macusani, Puno, durante la época de seca y las asociaciones con las variables edad y procedencia; así como establecer el promedio de carga parasitaria e identificar los géneros de helmintos presentes. Se colectaron muestras de heces de 1319 alpacas durante agosto a octubre de 2010 y se procesaron con las técnicas de flotación con solución Willis y sedimentación espontánea. Para la estimación de la carga e identificación de larvas de nematodos se utilizó el método McMaster modificado y Baermann, respectivamente. La prevalencia de helmintos fue de $63.9 \pm 2.6 \%$. En la prueba de regresión logística se determinó que la edad constituyó un factor de riesgo para la presencia de helmintos $(\mathrm{p}<0.05)$, donde alpacas de 5 meses a $<1$ año y aquellas de 1 a 3 años de edad presentaron 2.93 y 1.98 veces mayor riesgo de presentar esta parasitosis que animales > 3 años; en tanto que no hubo diferencias en prevalencias entre las dos comunidades. La carga parasitaria por cada género de nematodo no superó los 100 hpg. Los helmintos identificados fueron Nematodirus, Trichuris, Moniezia, Cooperia, Oesophagostomum, Trichostrongylus, Ostertagia, Bunostomum, Haemonchus, Capillaria y Lamanema, donde Nematodirus spp presentó una prevalencia del $52.8 \%$, seguido de Trichuris spp (10.8\%) y Moniezia $\operatorname{spp}(9.6 \%)$.

Palabras clave: alpaca, parásitos gastrointestinales, Puno, época seca

\section{Abstract}

The study aimed to estimate the prevalence of gastrointestinal helminth in alpacas of two peasant communities in Macusani district, Puno, Peru during the dry season and its association with age and origin; also, to establish the average parasite burden and to

${ }^{1}$ Laboratorio de Microbiología y Parasitología Veterinaria, ${ }^{2}$ Laboratorio de Reproducción Animal, ${ }^{3}$ Laboratorio de Medicina Veterinaria Preventiva, Facultad de Medicina Veterinaria, Universidad Nacional Mayor de San Marcos, Lima

${ }^{4}$ E-mail: achavezvg@gmail.com

Recibido: 29 de mayo de 2013

Aceptado para publicación: 24 de enero de 2014 


\begin{abstract}
identify the genus of helminth presents. Stool samples were collected from 1319 alpacas from August to October 2010 and were processed by the flotation method using Willis solution and by the spontaneous sedimentation technique. The parasite burden and identification of nematode larvae was done by the McMaster method and the modified Baermann technique respectively. The prevalence of helminths was $63.9 \pm 2.6 \%$. The logistic regression test showed that age was a risk factor for the presence of helminths $(\mathrm{p}<0.05)$, where alpacas of 5 months to $<1$ year old and those of 1 to 3 years old had 2.93 and 1.98 higher risk of this parasite in comparison to animals $>3$ years old, while there was no difference in the prevalence between the two communities. The parasite burden per each genus was lower than 100 epg. Helminth genus identified were Nematodirus, Trichuris, Moniezia, Cooperia, Oesophagostomum, Trichostrongylus, Ostertagia, Bunostomum, Haemonchus, Capillaria and Lamanema, where the prevalence of Nematodirus spp was the highest (52.8\%) followed by Trichuris spp (10.8\%) and Moniezia $\operatorname{spp}(9.6 \%)$.
\end{abstract}

Key words: alpaca, gastrointestinal parasites, Puno, dry season

\section{INTRODUCCIÓN}

El Perú es poseedor del mayor número de alpacas (Vicugna pacos) en el mundo y en el departamento de Puno se encuentran cerca de 2 millones de cabezas, que representan el $59 \%$ de la población nacional (CEPES, 2010). El 90\% de las alpacas se encuentra en manos de comunidades campesinas y pequeños productores, y es por ello que el distrito de Macusani, en la provincia de Carabaya, Puno, ubicado sobre los 4315 msnm, es considerado la «capital alpaquera del Perú y del mundo».

La crianza de alpacas es una actividad importante en las zonas de puna alta, donde estos animales son fuente de fibra, carne (Guerrero y Alva, 1986) y pieles útiles para el sustento de las familias campesinas; sin embargo, uno de los factores que afectan la productividad de los camélidos sudamericanos (CSA) son las enfermedades parasitarias (Ameghino y DeMartini, 1991).

Los nematodos ocasionan disminución del apetito, menor conversión alimenticia al generar competencia con el hospedero por los nutrientes, crecimiento deficiente, problemas de diarrea, etc. Asimismo, los cestodos, provocan en el hospedero una acción irritativa, mecánica (obstrucción intestinal) y tóxica que genera diversos tipos de enteritis según la carga parasitaria (Guerrero y Leguía, 1987). En consecuencia, los helmintos ocasionan en los CSA domésticos una disminución en la producción, tanto en carne como en fibra, además del decomiso de vísceras infectadas, que generan pérdidas que ascienden a cerca de 700 mil dólares anuales (Ministerio de Agricultura, 1973); además, del gasto adicional que implica el uso de antiparasitarios, lo que evidentemente va en detrimento de la economía de los productores (Rojas, 1990).

La edad del animal juega un rol importante en la infección parasitaria. Se conoce que alpacas menores de dos años son muy susceptibles a la infección por helmintos (Chávez et al., 1965; Guerrero y Alva, 1968; Dunn, 1983; Leguía y Casas, 1999; Bustinza, 2001). Otros factores de importancia son el pastoreo en áreas reducidas y sin una adecuada rotación de pasturas (Romero y Sanabria, 2005), y dentro del factor medioambiental se tiene que las cargas parasitarias disminuyen durante la época seca (Rojas, 1990; Leguía y Casas, 1999; Yucra, 2002).

La tasa de prevalencia de helmintos en alpacas de explotaciones medianas o grandes es alta, encontrándose en la literatura 
reportes que van desde 70 al 100\% (FAO, 2005). Sin embargo, son escasos los estudios realizados en pequeñas explotaciones o comunidades campesinas donde la crianza es generalmente mixta (Leguía y Casas, 1999). En el departamento de Puno se han reportado prevalencias parasitarias variables, siendo de $85 \%$ en los distritos de Mañazo y Cabanillas (CEDER, 2009) y de $38 \%$ en Quinsachata (Wolf, 2010).

Por lo tanto, el presente estudio tuvo como objetivo estimar la prevalencia de helmintos gastrointestinales en alpacas de dos comunidades del distrito de Macusani, Puno, durante la época de seca y las asociaciones con las variables edad y procedencia; asimismo, establecer el promedio de carga parasitaria e identificar los géneros de helmintos presentes.

\section{Materiales y Métodos}

\section{Lugar de Estudio}

El estudio se realizó en dos comunidades alpaqueras (Hatun Phinaya y Queracucho) de Macusani, provincia de Carabaya, departamento de Puno, a 4315 msnm, entre agosto y octubre de 2010. La crianza de animales era de tipo mixta, con alpacas, llamas y ovinos al pastoreo sobre pasturas naturales. La zona presenta un promedio anual de $672 \mathrm{~mm}$ de precipitación pluvial (SENAMHI, 2010) y una temperatura mínima y máxima de 3.9 y $5.2^{\circ} \mathrm{C}$, respectivamente. La época de seca se circunscribe a los meses de abril a noviembre y el resto del año a la época de lluvia.

\section{Tamaño de Muestra}

Para el cálculo del tamaño muestral se usó la fórmula de proporciones infinitas (Daniel, 1996), obteniendo un número mínimo de 364 alpacas por comunidad; no obstante, se evaluaron 598 y 721 alpacas de las comunidades Hatun Phinaya y Queracucho, respectivamente, dando un total de 1319 alpacas.

\section{Recolección de Muestras}

Se obtuvieron muestras fecales de manera aleatoria, directamente del recto, de alpacas Huacaya machos y hembras. Las muestras fueron clasificadas de acuerdo al grupo etario ( 5 meses a $<1$ año, de 1 a 3 años y $>3$ años). Se registró la fecha de muestreo, sexo, edad y lugar de procedencia. Las muestras fueron guardadas en recipientes térmicos con refrigerantes y trasladadas al Laboratorio de Parasitología de la Facultad de Medicina Veterinaria de la Universidad Nacional Mayor de San Marcos, Lima.

\section{Procesamiento y Análisis de Muestras}

En la evaluación coproparasitológica se utilizaron las técnicas cualitativas de flotación con solución Willis y de sedimentación espontánea. Asimismo, la técnica de McMaster modificada para la estimación de la carga parasitaria. Las muestras positivas a huevos tipo Strongylus fueron sometidas a cultivo de larvas, recuperándolas por el método de Baerman (Rojas, 2004). Los géneros de las larvas infectivas fueron determinados mediante características morfológicas y biométricas (Rojas, 1990; Leguía y Casas, 1999), previa inmovilización con solución yodo-yodurada (Rojas, 2004).

\section{Análisis Estadístico}

Se calculó la prevalencia de helmintos a partir de la proporción de muestras positivas, con los respectivos intervalos de confianza (Daniel, 1996). Asimismo, para la evaluación de las variables edad y procedencia como factores de riesgo para la infección por helmintos se utilizó la prueba de regresión logística (Daniel, 1996), empleando el software SPSS v. 17.0. 
Cuadro 1. Prevalencia de helmintos en alpacas Huacaya de dos comunidades del distrito de Macusani, Puno, mediante examen coproparasitológico (agosto-octubre, 2010)

\begin{tabular}{lccc}
\hline \multirow{2}{*}{ Variable } & $\mathrm{N}^{\mathrm{o}}$ alpacas & \multicolumn{2}{c}{ Positivas } \\
\cline { 3 - 4 } & & $\mathrm{n}$ & $\% \pm \mathrm{IC}^{1}$ \\
\hline \multicolumn{1}{l}{ Edad } & & & \\
$\quad$ 5 meses a $<1$ año & 310 & 241 & $77.7 \pm 4.6$ \\
$\quad$ 1 a 3 años & 520 & 355 & $68.3 \pm 3.9$ \\
$\quad$ >3 años & 489 & 247 & $50.5 \pm 4.4$ \\
Comunidad & & & \\
$\quad$ Hatun Phinaya & 598 & 363 & $60.7 \pm 3.9$ \\
$\quad$ Queracucho & 721 & 480 & $66.6 \pm 3.4$ \\
\hline Total & 1319 & 843 & $63.9 \pm 2.6$ \\
\hline Intervalo de confianza de 95\% & & &
\end{tabular}

\section{Resultados}

La prevalencia general de helmintos (nematodos y cestodos) en alpacas del distrito de Macusani mediante examen coproparasitológico fue de $63.9 \pm 2.6 \%$ (Cuadro 1). La variable edad constituyó un factor de riesgo para la presentación de la helmintiasis, siendo las alpacas de 5 meses a $<1$ año y aquellas de 1 a 3 años más susceptibles para la presentación de los helmintos $(\mathrm{p}<0.05)$, presentando 2.93 y 1.98 veces mayor riesgo de infección con respecto a la población etaria $>3$ años.

La carga parasitaria promedio en términos de huevos por gramos de heces (hpg) fue de 68.3 en Nematodirus spp, 52.3 en HTS, 51.4 en Trichuris spp, 54.2 en Capillaria spp y 50.0 hpg en Lamanema spp; es decir, ninguno superó los 100 hpg, considerándose estos valores como cargas bajas.

Se encontraron huevos de helmintos de los géneros Nematodirus, Trichuris, Capillaria, Lamanema y Moniezia, así como huevos tipo Strongylus (HTS), donde la mayor frecuen- cia fue para huevos de Nematodirus spp $(52.8 \%)$ y la menor para Lamanema spp $(0.7 \%)$. El único cestodo hallado fue Moniezia spp. Los animales menores del año de edad presentaron mayores prevalencias de Moniezia spp y Trichuris spp en comparación con los otros grupos etarios $(\mathrm{p}<0.05$; Cuadro 2).

En el cultivo de heces se identificaron los géneros de nematodos a través de la identificación de las larvas infectivas (L3) de los HTS. Se encontraron Cooperia spp (37\%), Oesophagostomum spp (23\%), Trichostrongylus spp (20\%), Ostertagia spp (14\%), Bunostomun spp (3\%) y Haemonchus spp (3\%).

\section{Discusión}

Estudios en la zona indican una gran variación de la prevalencia de helmintos en la alpaca; reportándose $69.7 \%$ de prevalencia en Puno, Ilave, Juli, Chucuito y Yunguyo, $33.8 \%$ en Huancané y Azángaro (Melo, 1997), $85 \%$ en el distrito de Mañazo, 
Cuadro 2. Prevalencia de huevos de helmintos en alpacas Huacaya de dos comunidades del distrito de Macusani, Puno (agosto-octubre, 2010)

\begin{tabular}{|c|c|c|c|c|c|c|c|}
\hline \multirow[b]{2}{*}{ Variable } & \multirow{2}{*}{$\begin{array}{l}\text { Alpacas } \\
\text { (n) }\end{array}$} & \multicolumn{6}{|c|}{ Helmintos } \\
\hline & & $\begin{array}{c}\text { Nematodirus } \\
(\%)\end{array}$ & $\begin{array}{l}\mathrm{HTS}^{1} \\
(\%)\end{array}$ & $\begin{array}{c}\text { Trichuris } \\
(\%)\end{array}$ & $\begin{array}{c}\text { Capillaria } \\
(\%)\end{array}$ & $\begin{array}{c}\text { Lamanema } \\
(\%)\end{array}$ & $\begin{array}{c}\text { Moniezia } \\
(\%)\end{array}$ \\
\hline \multicolumn{8}{|l|}{ Edad } \\
\hline $5 \mathrm{~m} a<1$ año & 310 & 54.2 & 5.5 & $24.5^{\mathrm{a}}$ & 3.2 & 0.6 & $28.4^{\mathrm{a}}$ \\
\hline 1 a 3 años & 520 & 60.2 & 2.3 & $9.4^{\mathrm{b}}$ & 1.7 & 0.8 & $5.2^{\mathrm{b}}$ \\
\hline$>3$ años & 489 & 44 & 7.4 & $3.7^{\mathrm{b}}$ & 1 & 0,6 & $2.2^{\mathrm{b}}$ \\
\hline \multicolumn{8}{|l|}{ Comunidad } \\
\hline $\begin{array}{l}\text { Hatun } \\
\text { Phinaya }\end{array}$ & 598 & 50.3 & 7.7 & 8.9 & 1.5 & 1.3 & 9.4 \\
\hline Queracucho & 721 & 54.8 & 2.6 & 12.5 & 2.1 & 0.1 & 9.7 \\
\hline Total & 1319 & 52.8 & 4.9 & 10.8 & 1.8 & 0.7 & 9.6 \\
\hline
\end{tabular}

Cabanillas (CEDER, 2009) y 38\% en Quinsachata (Wolf, 2010), mientras que en el presente estudio fue de $63.9 \pm 2.6 \%$.

Uno de los factores adicionales a los evaluados en el estudio que podrían haber influenciado la prevalencia de parasitismo en Puno serían las condiciones ambientales locales, principalmente la humedad y precipitación pluvial. Así, en el presente estudio en Macusani se tuvo $73.6 \%$ y $34.3 \mathrm{~mm}$, respectivamente, cifras muy superiores a las reportadas en el estudio de Quinsachata (55\% y $11.2 \mathrm{~mm}$, respectivamente; Wolf, 2010). La mayor humedad presente en Macusani favoreció el desarrollo y supervivencia parasitaria en el medio (Botero y Restrepo, 2003). Se conoce que la humedad superior al $70 \%$ permite el desarrollo de huevos de nematodos en pequeña escala, siendo de $96 \%$ la humedad ideal para su desarrollo (Cordero del Campillo et al., 1999).

Factores adicionales que afectan la frecuencia de parasitismo son el manejo animal y sanitario. Las dos comunidades en estudio realizan un sistema de explotación mixta donde alpacas, ovinos y llamas comparten las mismas pasturas contaminadas, lo cual unido a la baja especificidad de muchos helmintos, ocasionaría una infección cruzada (Soulsby, 1993), causando un mayor nivel parasitario (Keyyu et al., 2006). En el caso del estudio en la estación experimental del INIA (Instituto Nacional de Innovación Agraria) en Quinsachata (Wolf, 2010), se tuvo, por otro lado, un adecuado manejo animal y sanitario.

El mayor riesgo de infección con helmintos en alpacas jóvenes en relación a animales mayores de tres años se debe probablemente al efecto del destete, que coincide con la época seca, cuando los pastos son deficientes en cantidad y calidad, presentándose un estrés nutricional y una deficiente respuesta inmune de las alpacas frente a los parásitos (Leguía y Casas, 1999). Además, el pastoreo conjunto, donde animales adultos y crías conviven durante la lactación y el empadre, ocasiona la contaminación de los campos de pastoreo con niveles altos de larvas infectivas (Chávez et al., 1967). Esta si- 
tuación favorece que las crías ingieran larvas infectivas cuando inician el consumo de pasto desarrollando parasitismo gastrointestinal a temprana edad. Asimismo, cabe señalar que los animales viejos parasitados presentan un menor número de parásitos y estos tienden a ser más pequeños y menos fecundos que los presentes en animales jóvenes (Dunn, 1983).

Al no haber reportes establecidos sobre grados de infestación por helmintos en alpacas, se tomó como referencia los estudios en ovinos, donde se considera como carga moderada y severa recuentos de 1000 y $2000 \mathrm{hpg}$, respectivamente, en infecciones mixtas (Ueno y Goncalves, 1998). En base a esto, la carga promedio total mostrada en el estudio sería considerada leve, debido principalmente a las condiciones medio ambientales de la época seca; es decir, baja precipitación pluvial y cambios bruscos de temperaturas, factores que afectan seriamente el desarrollo y viabilidad de los estadios preparasíticos (Leguía y Bendezú, 1974).

La mayor evidencia parasitaria encontrada de helmintos correspondió a huevos de Nematodirus spp (52.8\%, Cuadro 2), concordando con otro estudio en la zona donde se halló una frecuencia de $69.7 \%$ de Nematodirus spp en alpacas (Melo, 1997). Los huevos de estos parásitos presentan una gran resistencia frente a la sequedad y bajas de temperatura, permitiendo el desarrollo larvario dentro del mismo. Los huevos de Nematodirus predominan sobre los HTS durante la época de sequía (Guerrero y Alva 1986; Leguía 1991; Leguía y Casas 1999; Eckert et al., 2005), siendo esta característica su mayor fortaleza para su supervivencia y alta frecuencia en el altiplano (Gorman, 1989).

La prevalencia de HTS fue baja (4.9\%) comparada a lo reportado por Melo (1997) en Huancané y Azángaro (33.4\%) y en otras localidades cercanas $(60.5 \%)$. La mayor presentación de este tipo de huevos se observa en la época lluviosa (Rojas, 1986; Quiroz, 2005), en tanto que cuando las condiciones ambientales son desfavorables suelen hacer hipobiosis, no eliminando huevos al medio ambiente (Leguía, 1991).

Lamanema chavezi es un parásito propio de los CSA, pero en el presente estudio se registró únicamente $0.7 \%$ de prevalencia, a diferencia otros reportes que indican una prevalencia de $28 \%$ en la zona de Huancané y Azángaro (Melo, 1997). La escasa precipitación pluvial durante los meses de muestreo pueden haber afectado los resultados, toda vez que Crofton (1963) considera que se necesita $50 \mathrm{~mm}$ de pluviosidad mensual para permitir el desarrollo del parásito en el medio ambiente. Asimismo, Rojas et al. (1981) demostraron que los huevos de Lamanema en época de sequía tardan de 20 a 40 semanas en desarrollarse.

La prevalencia general de Moniezia spp fue de $9.6 \%$, siendo los animales jóvenes los más afectados (28.4\%, Cuadro 2). Según Bustinza (2000), los animales menores de un año son los más susceptibles a cestodos, especialmente entre 3 a 4 meses y después del destete. Posteriormente, las alpacas adquieren una sólida inmunidad que limita la carga a 1 o 2 tenias por animal, aunque estas constituyen una fuente permanente de infección (Fernández, 1991).

El mayor porcentaje de larvas infectivas $\left(\mathrm{L}_{3}\right)$ procedentes de HTS correspondió al género Cooperia $(40 \%)$, concordando con Wolf (2010), a pesar de que este género se presenta generalmente en zonas templadas y cálidas (Romero y Sanabria, 2005), lo que indicaría que la humedad y aireación fueron las óptimas para su desarrollo. Estos son parásitos altamente prolíficos que producen de 1000 a 3000 huevos diarios (Boom y Sheath, 2008) y ocasionalmente realizan hipobiosis cuando la temperatura es extrema, lo que permite una mayor supervivencia (Soulsby, 1993; Fowler, 1998). Asimismo, las larvas infectivas pueden permanecer viables de 9 a 26 semanas (Boom y Sheath, 2008). 


\section{Conclusiones}

- La prevalencia de helmintos en alpacas del distrito Macusani, Puno, durante la época de seca fue de $63.9 \pm 2.6 \%$.

- La carga parasitaria de nematodos no superó los 100 huevos por gramo de heces, considerándose como carga parasitaria leve.

- Se identificaron parásitos de los géneros Nematodirus, Trichuris, Moniezia, Cooperia, Oesophagostomum, Trichostrongylus, Ostertagia, Bunostomum, Haemonchus, Capillaria y Lamanema.

\section{Agradecimiento}

Los autores agradecen a PROVÍAS por el financiamiento otorgado para el desarrollo del estudio.

\section{Literatura Citada}

1. Ameghino E, DeMartini J. 1991. Mortalidad crías de alpacas. Bol Div IVITA 24: 105-106.

2. Boom CJ, Sheath GW. 2008. Migration of gastrointestinal nematode larvae from cattle faecal pats onto grazable herbage. Vet Parasitol 157: 260-266.

3. Botero D, Restrepo M. 2003. Parasitosis humanas. Medellín: Corporación para Investigaciones Biológicas. $67 \mathrm{p}$.

4. Bustinza JA. 2000. Enfermedades de alpacas. $2^{\circ}$ ed. Arequipa: Universidad Nacional del Altiplano. $346 \mathrm{p}$.

5. Bustinza V. 2001. La alpaca. Puno: UNA. 480 p.

6. [CEDER] Centro de Estudios para el Desarrollo Regional. 2009. Desarrollo de las capacidades productivas y comerciales de los pequeños criadores de alpacas de los distritos de Mañazos y Cabanillas. Puno: CEDER. 28 p.
7. CEPES. 2010. Diagnóstico situacional de los camélidos en la región de Puno. [Internet], [4 mayo 2011]. Disponible en: http://www.cepes.org.pe/cendoc/ Propuesta_candidato_region_Puno.pdf

8. Chávez GC, Guerrero DC, Alva JM, Guerrero J. 1965. Parasites and parasitic diseases of Lama pacos (alpacas). Rev FMV-UNMSM 8: 1-4.

9. Chávez GC, Guerrero DC, Alva JM, Guerrero J. 1967. El parasitismo gastrointestinal en alpaca. Rev Fac Med Vet, Perú 21: 9-19.

10. Cordero del Campillo M, Rojo VF, Martínez FA, Sánchez AM, Hernández RS, Navarrete LC, et al. 1999. Parasitología veterinaria. Madrid: McGraw-Hill. 990 p.

11. Crofton HD. 1963. Nematode parasite population in sheep and pasture. UK: Commonwealth Agricultural Bureaux. $104 \mathrm{p}$.

12. Daniel D. 1996. Bioestadística base para el análisis de las ciencias de la salud. $5^{\circ}$ ed. México: Limusa. 480 p.

13. Dunn AM. 1983. Helmintologia veterinaria. $2^{\circ}$ ed. México: Manual Moderno. $1832 \mathrm{p}$.

14. Eckert J, Friedhoff H, Zahner P, Deplazes. 2005. Parasitología para la Medicina Veterinaria. Alemania: Enke Verlag Stuttgart. $218 \mathrm{p}$.

15. FAO. 2005. Proyecto de cooperación técnica en apoyo a la crianza y aprovechamiento de los camélidos sudamericanos en la región andina. 62 p. [Internet], [4 mayo 2011]. Disponible en: http:// www.rlc.fao.org/es/ganaderia/pdf/ 2914per.pdf

16. Fernández B. 1991. Avances y perspectivas del conocimiento de los camélidos sudamericanos. Chile: Oficina Regional FAO para América Latina. $325 \mathrm{p}$.

17. Fowler ME. 1998. Medicine and surgery of South American camelids (llama, alpaca, vicuña, guanaco). $2^{\text {nd }}$ ed. Iowa, USA: Iowa State University Press. 549 p. 
18. Gorman T. 1989. Tópicos sobre la biología y manejo de los camélidos sudamericanos. Chile: Facultad de Ciencias Veterinarias y Pecuarias. $16 \mathrm{p}$.

19. Guerrero C, Alva J. 1986. Gastroenteritis nematódica y sarna en alpacas. Bol IVITA UNMSM 21:25-33.

20. Guerrero C, Leguía G. 1987. Enfermedades infecciosas y parasitarias de alpacas. Rev Cam Sudamer UNMSMIVITA 4: 32-82.

21. Guerrero DC, Alva MJ. 1968. Algunos aspectos epidemiológicos de la gastroenteritis verminosa en las alpacas. Bol IVITA UNMSM 3: 54-55.

22. Keyyu JD, Kassuku AA, Msalilwa LP, Monrad J, Kyvsgaard NC. 2006. Cross sectional prevalence of helminth infections in cattle on traditional, small scale and large scale dairy farms in Iringa District, Tanzania. Vet Res Commun 30: 45-55.

23. Leguía P. 1991. Enfermedades parasitarias. Lima: Ed de Mar. 190 p.

24. Leguía P, Bendezú B. 1974. Observaciones de campo sobre la epidemiología de la gastroenteritis verminosa en alpacas (Lama pacos) de Cerro de Pasco. Rev Inv Pec IVITA 3: 3-7.

25. Leguía P, Casas E. 1999. Enfermedades parasitarias y atlas parasitológico de camélidos sudamericanos. Lima: Ed de Mar. $190 \mathrm{p}$.

26. Melo A. 1997. Sistemas de control y manejo sanitario de las alpacas y llamas en la región andina del sur peruano. Rev FMVZ-UNA, Puno 1: 54-59.

27. Ministerio de Agricultura. 1973. Estudio de la evaluación de problemas de carnes en el Perú. Tomo V. Lima: MINAG. $140 \mathrm{p}$.

28. Quiroz H. 2005. Parasitología y enfermedades parasitarias en animales domésticos. México: Limusa. 827 p.

29. Rojas CM. 1986. Bases para la prevención de la nematodiasis gastroentérica de las alpacas. Rev Cam Sudamer UNMSM-IVITA 3: 5-8.
30. Rojas CM. 1990. Parasitismo de los rumiantes domésticos: terapia, prevención y modelos para su aprendizaje. Lima: Ed Maijosa. 383 p.

31. Rojas CM. 2004. Nosoparasitosis de los rumiantes domésticos peruanos. $2^{\circ} \mathrm{ed}$. Lima: Ed Maijosa. $146 \mathrm{p}$.

32. Rojas M, Nuñez A, Alva J. 1981. Observación del desarrollo y sobrevivencia de Lamanema chavezi en condiciones naturales. Rev Cam Sudamer UNMSMIVITA 2: 34-38.

33. Romero J, Venturini L, Vignau M. 2005. Parasitología práctica y modelos de enfermedades parasitarias. En: Romero J, Sanabria R (eds). Parasitismo gastrointestinal y pulmonar de rumiantes. Argentina: Universidad Nacional de la Plata. p 100-103.

34. [SENAMHI] Servicio Nacional de Metereología e Hidrología 2010. Oficina de Estadística e Informática. Estación Metereológica de Macusani, Puno. [Internet], [4 mayo 2011]. Disponible en: http://www.senamhi.gob.pe/include_mapas/_dat_esta_tipo.php? estaciones $=000777$

35. Soulsby EJL. 1993. Parasitología y enfermedades parasitarias en los animales domésticos. $7^{\circ}$ ed. México: Interamericana. $820 \mathrm{p}$.

36. Ueno H, Goncalves PC. 1998. Manual para diagnostico das helmintoses de ruminantes. $4^{\circ}$ ed. Brasil: Salvador de Bahia. $145 \mathrm{p}$.

37. Wolf D. 2010. Untersuchungen zur Seroprävalenz von zystenbildenden Kokzidien und $\mathrm{zu}$ Gastrointestinalparasitosen bei Neuweltkameliden in Peru. Deutschland: Fachbereich Veterinärmedizin der Justus-LiebigUniversität Gießen. 154 p.

38. Yucra D. 2002. Carga parasitaria gastrointestinal, lesiones anatomopa-tológicas, respuesta celular y patrón humoral en alpacas de una comunidad campesinaPuno. Tesis de Maestría. Lima: Univ Nacional Mayor de San Marcos. 43 p. 